\title{
High mobility group box-1 in hypothalamic paraventricular nuclei attenuates sympathetic tone in rats at post-myocardial infarction
}

\author{
Pang Li, Yin Jie, Shi YuGen, Wang Yu, Suhua Yan \\ Department of Cardiology, Shandong Provincial Qianfoshan Hospital, \\ Shandong University, Jinan, China
}

\begin{abstract}
Background: Inflammation is associated with increased sympathetic drive in cardiovascular diseases. The paraventricular nucleus (PVN) of the hypothalamus is a key regulator of sympathetic nerve activity at post-myocardial infarction (MI). High mobility group box-1 (HMGB1) exhibits inflammatory cytokine-like activity in the extracellular space. Inflammation is associated with increased sympathetic drive in cardiovscular diseases. However, the role of HMGB1 in sympathetic nerve activity at post-MI remains unknown. The aim of the present study is to determine the role and mechanism of HMGB1 in the PVN, in terms of sympathetic activity and arrhythmia after MI.

Methods: Sprague-Dawley rats underwent left anterior descending coronary artery ligation to induce MI. Anti-HMGB1 polyclonal antibody or control IgG was bilaterally microinjected into the PVN (5 $\mu L$ every second day for seven consecutive days). Then, renal sympathetic nerve activity (RSNA) was recorded. The association between ventricular arrhythmias (VAs) and MI was evaluated using programmed electrophysiological stimulation. After performing electrophysiological experiments in vivo, immunohistochemistry was used to detect the distribution of HMGB1, while Western blot was used to detect the expression of HMGB1 and $p-E R K$ in the PVN of MI rats.

Results: HMGB1 and p-ERK were upregulated in the PVN in rats at post-MI. Moreover, bilateral PVN microinjection of anti-HMGB1 polyclonal antibody reversed the expression of $H M G B 1$ and $p-E R K$, and consequently decreased the baseline RSNA and inducible VAs, when compared to those in sham rats.

Conclusions: These results suggest that MI causes the translocation of HMGB1 in the PVN, which leads to sympathetic overactivation through the ERK1/2 signaling pathway. The bilateral PVN microinjection of anti-HMGB1 antibody can be an effective therapy for MI-induced arrhythmia. (Cardiol J 2019; 26, 5: 555-563)
\end{abstract}

Key words: HMGB1, ERK, hypothalamic paraventricular nucleus, sympathetic nerve, myocardial infarction

\section{Introduction}

Increasingly more attention has been given to the role of proinflammatory cytokines in cardiovascular and cardiovascular-related injuries.

High-mobility group box 1 (HMGB1) is a member of the HMGB protein family, which is a highly conserved non-histone nuclear protein in- volved in damage response. HMGB1 was a critical co-factor of somatic cell transcriptional regulation [1]. It plays roles in a wide variety of processes, including inflammation, immune responses, apoptosis and responses to injury [2, 3]. Furthermore, HMGB1 can be passively released from damaged cells to exacerbate inflammation through receptors, including the receptor for advanced glyca-

Address for correspondence: Dr. Suhua Yan, Department of Cardiology, Shandong Provincial Qianfoshan Hospital, Shandong University 250014, Jinan, China, tel: +86 531 89268550, fax: +86 531 82963647, e-mail: yansuhua296@163.com 
tion end products (RAGE) and toll-like receptors (TLR2 and TLR4) [4-6]. These receptors activate multiple intracellular signaling pathways, including mitogen-activated protein kinases (MAPKs), extracellular signal-regulated kinase (ERK)1/2, and phosphatidylinositol-4,5-bisphosphate 3-kinase (PI3K)/protein kinase B (AKT). Phosphorylated (p-) ERK1/2 has numerous cytoplasmic and nuclear effects [7, 8]. Among its nuclear effects, p-ERK1/2 can activate multiple nuclear transcription factors [7-10], and its products may include RAS components and inflammatory mediators $[9,11,12]$.

The paraventricular nucleus (PVN) is an integrative site in regulating sympathetic outflow and cardiovascular activity [13, 14]. Previous studies have shown that PVN is involved in excessive sympathetic activation and enhanced cardiac sympathetic afferent reflex in chronic heart failure [15-19]. However, it remains unknown how inflammatory mediators form in the PVN and enhance sympathetic nervous system activity in acute myocardial infarction (AMI) rats. In the present study, a putative mechanism was examined. It was determined whether myocardial infarction (MI) could lead to HMGB1 release and ERK1/2 MAPK signaling activation in the PVN, thereby contributing to sympathetic activation. The present results indicate that HMGB1 and ERK1/2 MAPK signaling contribute to its generation and sympathetic activation, which therefore can be reduced by HMGB1 antagonists. These findings show that this can be a potential target for AMI therapy.

\section{Methods}

\section{Animals}

All experimental procedures were conducted in accordance with the Shandong University Institutional Animal Care and Use Committee guidelines for animal experiments, and were approved by the University's Committee. Adult male Sprague-Dawley rats (Charles River, Beijing, China), weighing 250-280 g, were used for all experiments. These rats were housed in a temperature-controlled room $\left(23 \pm 2^{\circ} \mathrm{C}\right)$ with light-controlled animal quarters, and were provided free access to laboratory chow and water.

\section{Location and catheterization of the PVN}

Sixty rats were anesthetized via intraperitoneal injection of chloral hydrate $(40 \mathrm{mg} / \mathrm{kg})$. Then, the rats were fixed on the brain stereotactic locator (RWD Life Science Co., Shenzhen, China), a skin incision was performed along the sagittal suture to expose the skull, and the anterior and posterior fontanelles were adjusted to the same level. The stereotaxic coordinates for the PVN were $1.8 \mathrm{~mm}$ caudal from the bregma, $0.4 \mathrm{~mm}$ lateral to the midline and $7.9 \mathrm{~mm}$ ventral to the dorsal surface. A stainless-steel casing with a core outer diameter of $0.6 \mathrm{~mm}$ and an inner diameter of $0.4 \mathrm{~mm}$ (RWD Life Science Co., Shenzhen, China) was implanted into the skull using a gripper instrument (RWD Life Science Co., Shenzhen, China). The cannula was fixed with dental cement, and penicillin powder was sprayed on the top of the skull. Then, the skin incision was sutured. Rats were fed in a single cage. After 1 week of recovery, 49 rats implanted with a cannula survived for further testing.

\section{The MI model}

The 49 rats that were successfully implanted with a cannula were anesthetized via intraperitoneal injection of chloral hydrate $(40 \mathrm{mg} / \mathrm{kg})$. The animals underwent a thoracotomy and pericardiotomy, and the left anterior descending coronary artery was ligated to establish the MI, as previously reported [20]. Sham rats underwent a thoracotomy and pericardiotomy without coronary artery ligation. Rats underwent electrocardiography (ECG) monitoring using an animal biological function experiment system (BL-420S, TaiMeng, China) during the MI surgery. The infarction was confirmed by ST segment elevation, regional cyanosis and wall motion abnormalities. The ST segment (from the end of the QRS wave to the beginning of the $T$ wave) elevation after ligation of coronary artery is one of the evaluation of the MI model (Fig. 1A). With respect to clinical importance, only rats with moderate infarct size (30-50\%) were enrolled.

\section{PVN microinjection}

Rats received bilateral PVN microinjections of chicken anti-HMGB1 polyclonal antibody (ShinoTest Corporation, Tokyo, Japan; $10 \mu \mathrm{g}$ in $10 \mu \mathrm{L}$ of $10 \mathrm{mM}$ of Tris-buffered saline, $\mathrm{pH}$ 7.4, $5 \mu \mathrm{L}$ every second day for seven consecutive days), a dose of anti-HMGB1 polyclonal Ab similar to that used in previous studies, or an equivalent volume of control IgG. The PE pipe with a length of $15-20 \mathrm{~cm}$ and the casing pipe with a diameter of $0.4 \mathrm{~mm}$ were connected, and these were subsequently connected to a microsyringe. The bilateral PVN microinjections were carried out using a micropump injection device (RWD Life Science Co., Shenzhen, China). The bilateral PVN microinjection volume was $5 \mu \mathrm{L}$ for each site. The injection rate was $0.5 \mu \mathrm{L} /$ $/ \mathrm{min}$. After the injection, the syringe was left for 


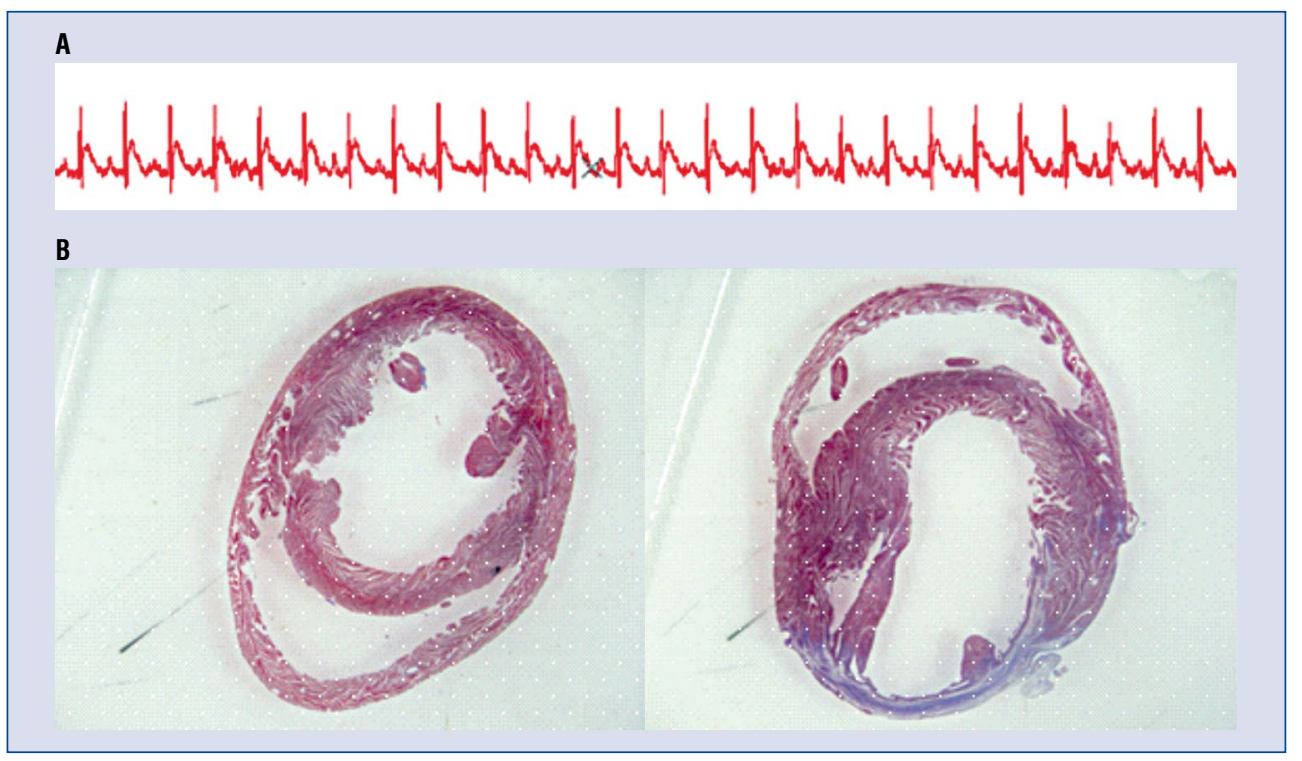

Figure 1. Evaluation of the myocardial infarction (MI) model; A. Electrocardiogram before (up) and after (down) ligation of coronary artery. The ST segment elevated after ligation of coronary artery; B. Representative histologic image of the heart stained with Massion's trichrome. Sections from the sham operation (left) and MI (right) rat hearts, respectively. Myocytes are red and fibrotic tissues are blue.

an additional 5 min before it was slowly retracted. At the end of the experiment, $50 \mathrm{~nL}$ of Evans Blue (2\%) was injected into the microinjection site for histological identification. Only the data from rats, in which the microinjection sites were within the boundaries of the PVN were used for analysis. Rats with microinjection sites outside the PVN or at the margin of the PVN were excluded from the data analysis.

\section{Electrophysiological experiments}

After 7 days, the rats were anesthetized, as previously described. Then, a second thoracotomy was performed. The protocol for programmed electrophysiological stimulation was similar to that described in a previous study of the investigators [21]. Programmed electrical stimulation was performed to measure the ventricular effective refractory period (VERP), while inducing ventricular arrhythmias (VAs). Briefly, the stimulation intensity was twice the threshold, and stimulus length was $5 \mathrm{~ms}$. In order to induce VAs, the pacing involved a cycle length of $180 \mathrm{~ms}\left(\mathrm{~S}_{0}\right)$, followed by $1-3$ extrastimuli $\left(\mathrm{S}_{1}, \mathrm{~S}_{2}\right.$ and $\left.\mathrm{S}_{3}\right)$ at shorter coupling intervals. In order to determine the VERP, a single extrastimulus was introduced at progressively shorter intervals. The VERP was longest at the $\mathrm{S}_{1}-\mathrm{S}_{2}$ interval, which did not evoke a premature ventricular depolarization. The experiment was typically completed within $10 \mathrm{~min}$. The results were classified as follows:
(1) inducible sustained monomorphic ventricular tachycardia (MVT); (2) inducible polymorphic ventricular tachycardia (PVT), and ventricular fibrillation (VF); (3) no inducible ventricular tachycardia VT/VF. All rats underwent 6-lead ECG.

\section{RSNA recording and measurement}

A retroperitoneal incision was made for the isolation of the left renal sympathetic nerve. The nerve was cut distally to eliminate its afferent activity, and placed on a pair of silver electrodes, which were immersed in warm mineral oil. The renal sympathetic nerve activity (RSNA) was amplified with a four channel AC/DC differential amplifier (DP-304; Warner Instruments, Hamden, CT, USA) with a high pass filter at $100 \mathrm{~Hz}$ and a low pass filter at $3000 \mathrm{~Hz}$. The RSNA was integrated at a time constant of $100 \mathrm{~ms}$. Background noise was determined, as previously reported [22]. Basal nerve activity (baseline) was determined by efferent RSNA at the beginning of the experiment. The RSNA activity during the experiment was calculated by subtracting the background noise from the recorded value. The RSNA responses were expressed as a percentage change from the basal value.

\section{Heart tissue preparation}

After the electrophysiological study, rats were sacrificed, and the hearts were rapidly removed. 
A sample of fresh cardiac tissue was immersed in $10 \%$ formalin for $24 \mathrm{~h}$, embedded in paraffin, cut into $10-\mu \mathrm{m}$ sections, and stained with Masson's trichrome (Fig. 1B). Then, corresponding heart positions were sampled in sham rats. Sections from sham group (Fig. 1A), MI group (Fig. 1B). Myocytes are red, and fibrotic tissues are blue.

\section{ELISA}

A rat noradrenaline (NA) ELISA Kit (Catalog Number CSB-E07022r, CUSABIO) was used to detect serum NE concentrations, according to manufacturer instructions. The intra- and intersample variability for each kit was $<8 \%$.

\section{Western blot analysis}

The expression of HMGB1 and p-ERK in the PVN were detected by Western blot analysis. For immunoblot analyses, proteins were isolated using a protein extraction kit (Beyotime Institute of Biotechnology, Jiangsu, China). The extracted protein was measured using a BCA protein assay reagent kit (Pierce, Madison, WI, USA). Equal amounts of total protein ( $80 \mu \mathrm{g}$ of protein/lane) were resolved on $5-10 \%$ SDS-PAGE gels, and transferred onto polyvinylidene difluoride membranes. Then, the membranes were blocked with 5\% non-fat dry milk in phosphate buffered saline (PBS) containing $0.05 \%$ Tween 20 , and incubated overnight at $4{ }^{\circ} \mathrm{C}$ with the respective primary antibodies against HMGB1 (ab172730-Rabbit monoclonal IgG; 1:20,000; Abcam) and primary antibodies against p-ERK (1:2,000; 9101; Cell Signaling Technology). Next, the membranes were incubated with horseradish peroxidase-conjugated goat anti-mouse or anti-rabbit secondary antibodies (1:10,000; Zhongshan Golden Bridge Biotechnology). The blots were developed using an enhanced chemiluminescence detection kit (Millipore, Billerica, MA), and visualized using a FluorChem E Imager (Protein-Simple, Santa Clara, CA). The densities relative to $\beta$-actin were analyzed using NIH ImageJ software.

\section{Immunohistochemistry}

After the electrophysiology studies, the brain was rapidly removed, and samples were immersed in $10 \%$ formalin for $24 \mathrm{~h}$, embedded in paraffin and cut into $5-\mu \mathrm{m}$ thick slices. The paraffin sections were deparaffinized, rehydrated and soaked in $0.1 \mathrm{M}$ of citric acid buffer for $15 \mathrm{~min}$ at $92-98^{\circ} \mathrm{C}$ in a microwave oven, and washed with PBS. Then, the sections were incubated with the primary antibodies of anti-HMGB1 (ab172730-Rabbit monoclonal IgG,
1:5,000; Abcam) overnight at $4^{\circ} \mathrm{C}$. Subsequently, the samples were incubated with horseradish peroxidase-conjugated secondary antibodies of rabbit anti-sheep IgG (KPL, Gaithersburg, MD, USA) and goat anti-mouse IgG (Zhongshan Golden Bridge Biotechnology) for $1 \mathrm{~h}$ at $37^{\circ} \mathrm{C}$. Immunore-activity was developed with 3,3'-diaminobenzidine tetrahydrochloride (Zhongshan Golden Bridge Biotechnology). Finally, the sections were counterstained with hematoxylin, mounted and examined by microscopy.

\section{Statistical analysis}

All data were expressed as mean \pm standard deviation (SD). The significance of differences in mean values was analyzed by the unpaired t-test. Analyses were performed using SPSS 17.0 software (SPSS Inc., Chicago, IL, USA). The differences were considered significant at $\mathrm{p}<0.001$.

\section{Results}

\section{The expression of HMGB1 in the PVN}

Using immunohistochemistry, the expression of HMGB1 in the PVN of the MI group and MI+Anti-HMGB1 polyclonal antibody group were measured. Few HMGB1 appeared in control and sham-operated PVN (Figs. 2A, B). Compared with sham-operated PVN, MI increased the density of HMGB1-positive nucleus in the PVN (Fig. 2C). In addition, density of HMGB1-positive nucleus was lower in MI PVN with anti-HMGB1 polyclonal antibody treatment, than in MI alone (Fig. 2D).

\section{Effects of anti-HMGB1 polyclonal antibody} on the expression of HMGB1 and p-ERK in the paraventricular nucleus

HMGB1 levels in the PVN were upregulated in MI rats, when compared with sham-operated rats ( $p<0.001$, Fig. 1). Moreover, HMGB1 levels were lower in MI with MI+Anti-HMGB1 polyclonal antibody, than in MI alone ( $\mathrm{p}<0.001$, Fig. 1$)$.

The nuclear protein levels of $\mathrm{p}$-ERK in the PVN were higher in rats with MI, than in shamoperated rats $(p<0.001$, Fig. 1$)$. Moreover, antiHMGB1 polyclonal antibody treatment significantly reduced the MI-induced nuclear expression of p-ERK (p $<0.001$, Fig. 3).

\section{Effects of anti-HMGB1 polyclonal antibody on baseline RSNA}

Anti-HMGB1 polyclonal antibody ( $5 \mu \mathrm{L}$, qod, 7 consecutive days) was microinjected into the PVN after MI, and RSNA was expressed as a percentage 


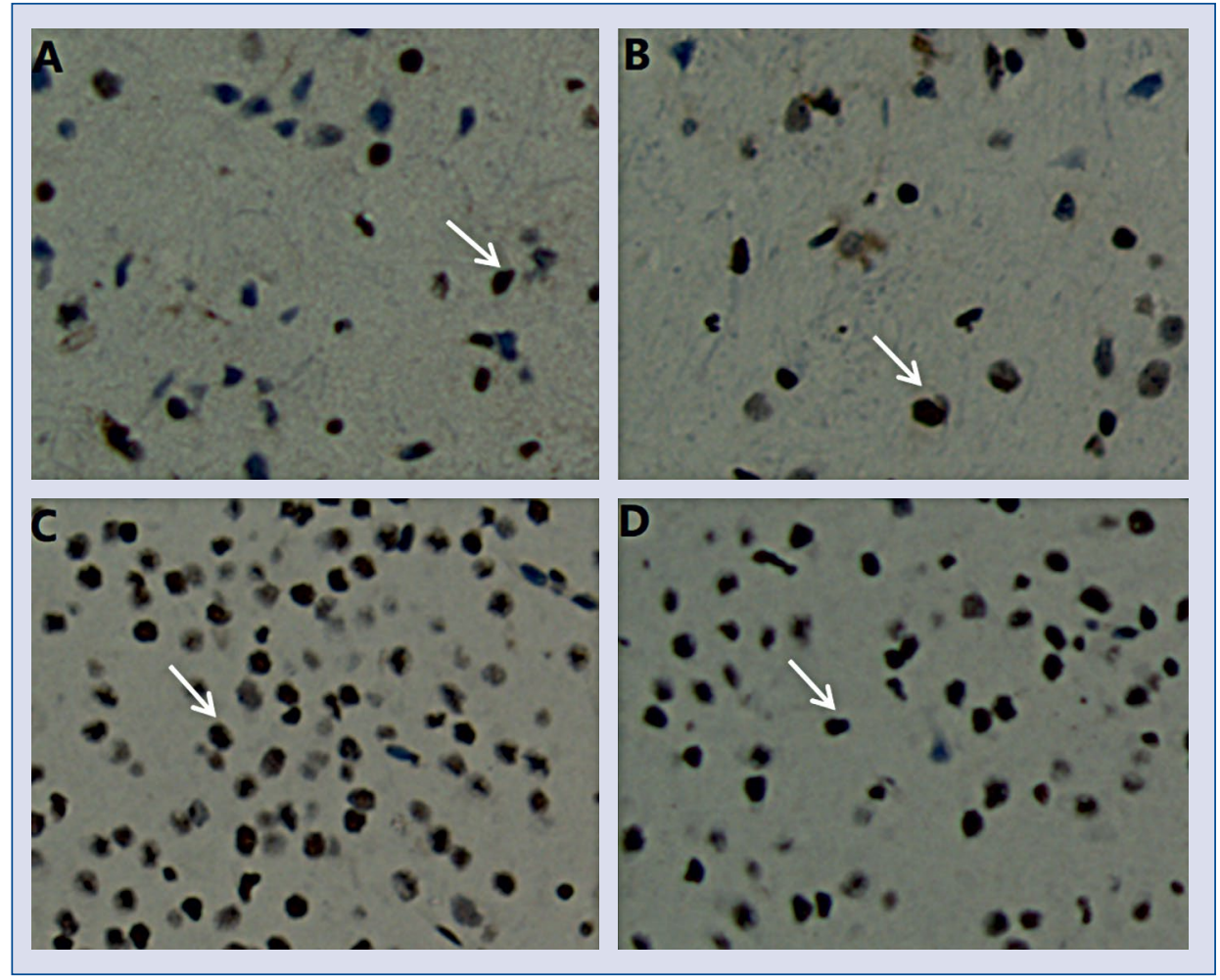

Figure 2. A-D. Representative immunostaining of high mobility group box-1 (HMGB1) in the paraventricular nucleus from sham-operated, myocardial infarction (MI), and MI+Anti-HMGB1 polyclonal antibody treated rats (magnification, $\times 400$ ). Brown represents the positive staining (arrows). Bar $=100 \mu \mathrm{m}$.

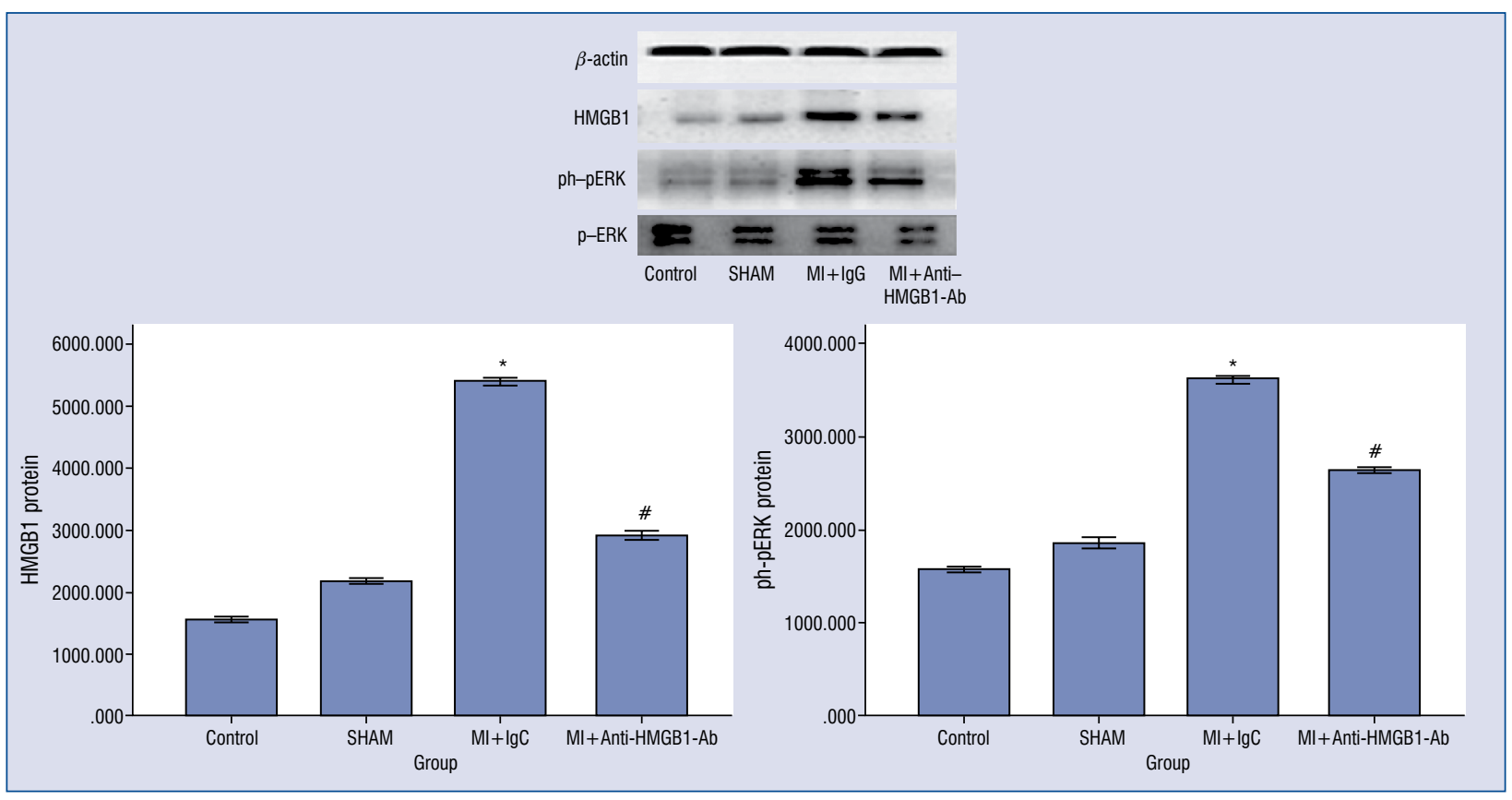

Figure 3. Western blot analysis of the protein levels of high mobility group box-1 (HMGB1) (25 kDa) and ph-pERK $(42 \mathrm{kDa})$ in cytoplasmic protein homogenates of the paraventricular nucleus. The quantification is relative to $\beta$-actin levels. Data are presented as mean \pm standard deviation; ${ }^{*} \mathrm{p}<0.001$ vs. sham; ${ }^{*} \mathrm{p}<0.001 \mathrm{vs}$. MI+Anti-HMGB1 polyclonal antibody. 


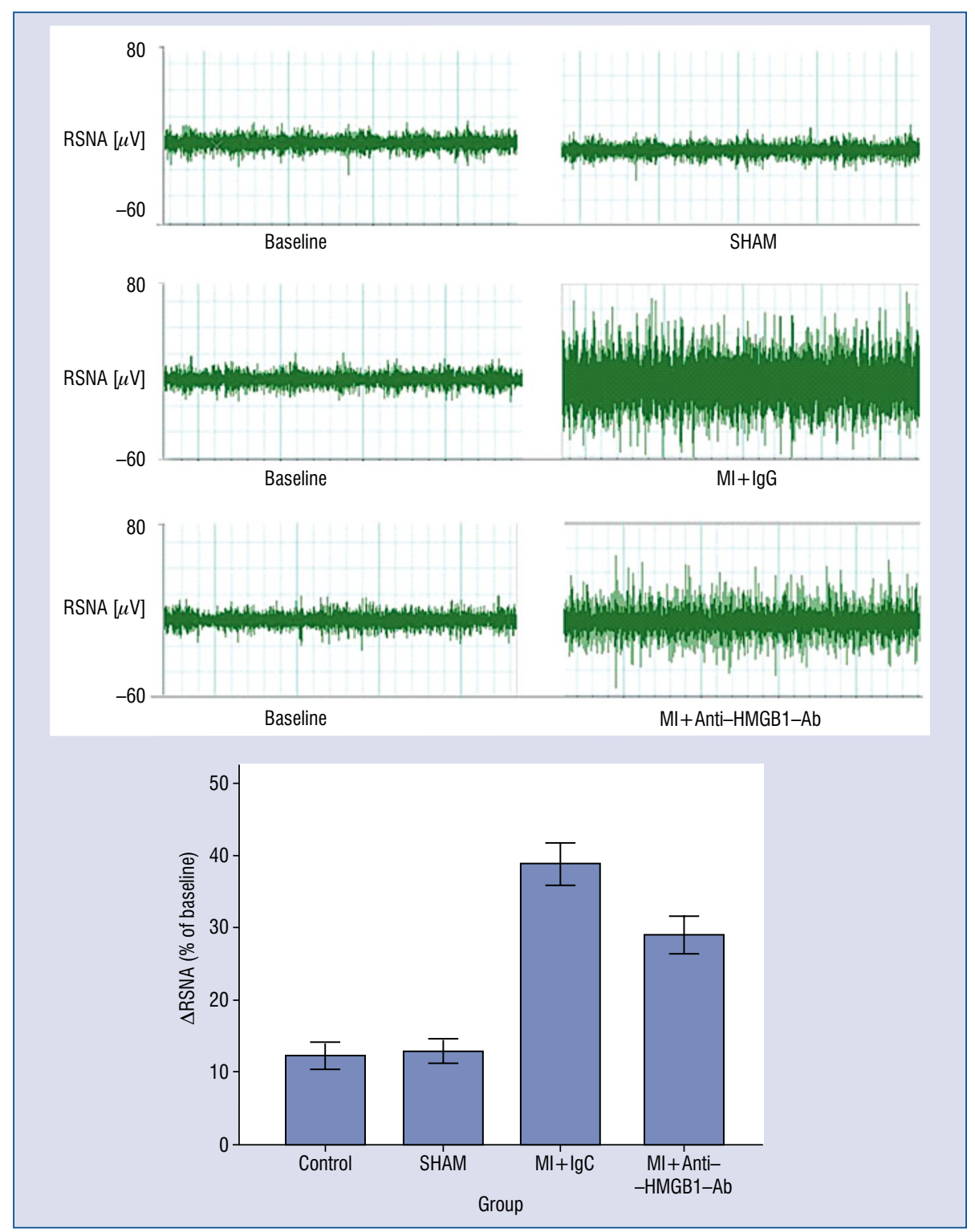

Figure 4. The segments of the original recordings obtained from individual rats in each experimental group show the responses of the renal sympathetic nerve activity (RSNA). Mean changes in RSNA after the microinjection of anti-HMGB1 polyclonal antibody into the paraventricular nucleus vs. the sham group; $\mathrm{p}<0.001 ; \mathrm{MI}+\mathrm{Anti-HMGB1}$ polyclonal antibody treatment group vs. the MI group; $\mathrm{p}<0.001$.

change from the basal value. As shown in Figure 4, compared with the AMI group, RSNA responses decreased in the MI+Anti-HMGB1 polyclonal antibody groups.

\section{Serum content of norepinephrine}

In order to further examine the action of sympathetic activity, norepinephrine (NE) levels in serum were measured by ELISA. As shown in Figure 5, the NE level in MI+Anti-HMGB1 polyclonal antibody rats was higher than in rats in the control group $(17.101 \pm 0.490$ vs. $14.949 \pm 0.562$; $\mathrm{p}<0.001)$, and was lower in MI rats $(21.047 \pm$ $\pm 1.358 \mathrm{pg} / \mathrm{mL} ; \mathrm{p}<0.001)$.

\section{Electrophysiological characterization}

In order to further elucidate an association of VAs and MI in rats, programmed electrophysiological stimulation was performed. No rat experienced spontaneous VAs during the placement of the electrodes, and none of the rats died during the electrophysiological study. VAs occurred in $1 / 10(10.0 \%)$ rats in the control group, which was significantly fewer than those in the MI+Anti- 


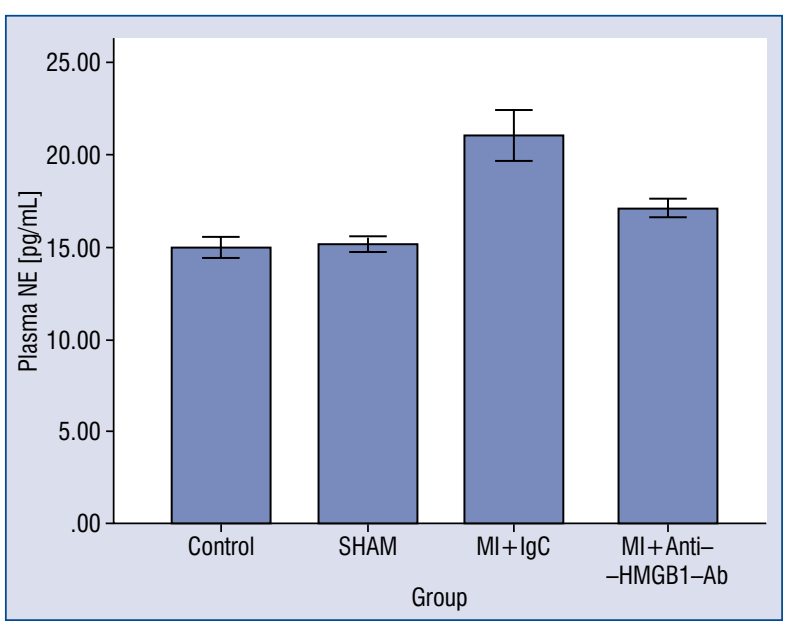

Figure 5. Norepinephrine content in serum of rats. ELI$\mathrm{SA}$ results of the NE content in serum obtained from rats in the control group, myocardial infarction (MI) group and $\mathrm{MI}+$ Anti-HMGB1 polyclonal antibody group. Data are expressed as mean \pm standard deviation.

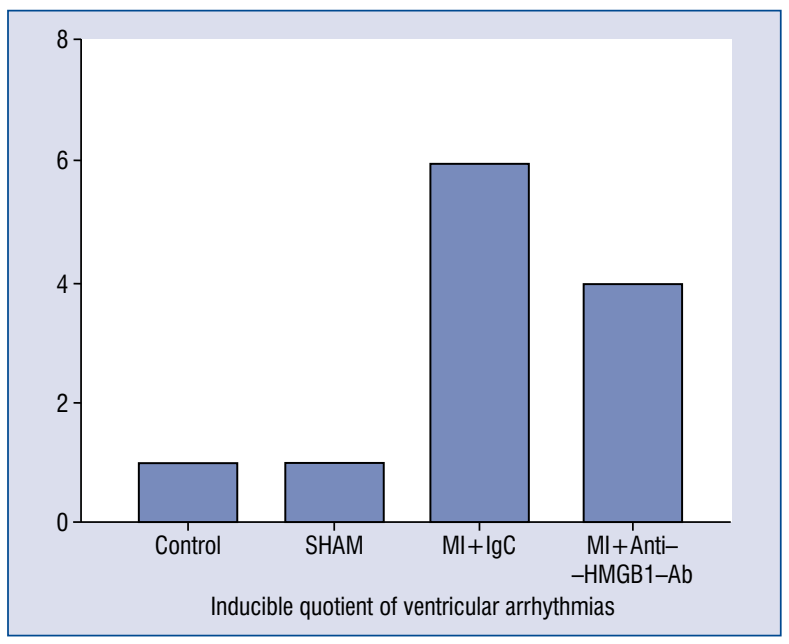

Figure 6. Comparisons of the arrhythmia score between the four groups at 7 days post-myocardial infarction (MI).

-HMGB1 polyclonal antibody $(4 / 11,36.4 \%)$ and MI $(6 / 12,50 \%)$ groups. The recordings of inducible VAs are presented in Figure 6.

\section{Discussion}

According to available research, the present study was the first to explore the role and mechanism of HMGB1 in the PVN in the development of sympathetic overaction and ventricular arrhythmia in MI rats.

There are several mechanisms between peripheral sympathetic and ventricular arrhythmo- genesis. The PVN of the hypothalamus is a critical site of autonomic and neuroendocrine regulation [23]. Inflammation is associated with increased sympathetic drive in cardiovascular diseases. In particular, recent studies have established a causal relationship between inflammation and the activation of the sympathetic nervous system [24, 25]. However, the mechanism of inflammatory mediators in sympathetic overactivity remains unknown. HMGB1 is normally found in the nucleus. When cell damage occurs, HMGB1 is translocated to the cytoplasm and released by the cell to act as a multifunctional cytokine with roles in infection, organ dysfunction, inflammation, and immune responses $[26,27]$. Consistently, it was found that the expression of HMGB1 significantly increased in the PVN after MI in rats. The significant increase in HMGB1 levels in the PVN after MI and the HMGB1 expression demonstrated by immunohistochemistry support the finding that a considerable amount of HMGB1 was released into the extracellular space.

Recent studies have revealed that the pharmacological manipulation of neuronal activity within the PVN can markedly alter sympathetic nerve activity [28]. Therefore, the investigators determined the effect of HMGB1 in the PVN on sympathetic activity and arrhythmia after MI, as well as its possible mechanism through the bilateral PVN microinjection of anti-HMGB1 polyclonal antibody, which is an HMGB1 antagonist.

One novel finding in the present study was that levels of HMGB1 and p-ERK were significantly elevated in the PVN, which was accompanied by high RSNA and high-risk VAs, in rats after MI.

In addition, another new finding in the present study was that the administration of anti-HMGB1 polyclonal antibody in the PVN effectively inhibited the expression of HMGB1 and p-ERK in MI rats. In addition, it was found that the administration of antiHMGB1 polyclonal antibody in the PVN prevented an increase in RSNA, and reduced a high risk of VAs in MI rats. Therefore, it was speculated that HMGB1 in the PVN after $\mathrm{MI}$ in rats may result in sympathetic overaction through the ERK1/2 signaling pathway.

There are at least two mechanisms by which ERK1/2 signaling might contribute to sympathetic excitation. Once activated, p-ERK1/2 can stimulate several transcription factors, such as activator-protein 1, Elk-1, nuclear factor kappa B and cyclic AMP response element-binding protein [7-10], in which the downstream products may include key excitatory elements, such as angiotensinogen [29], the precursor of ANG II, $\mathrm{AT}_{1} \mathrm{R}[11,12]$, tumor necrosis factor $\alpha$ and interleukin- $1 \beta[9,30,31]$, and COX-2 [32], 
the inducible enzyme that produces $\mathrm{PGE}_{2}$. A second mechanism by which ERK1/2 signaling may contribute to increased sympathetic activation is by disinhibiting presympathetic neurons [33].

In summary, HMGB1 in the PVN in rats after MI regulate RSNA through ERK1/2 signaling, which may well-contribute to generation of excitatory and inflammatory mediators in the PVN in MI rats. This is an important mechanism in sympathetic activation and VAs in rats after MI.

\section{Conclusions}

Overall, these results indicate that the expression of HMGB1 in the PVN in MI rats and ERK1/2 signaling may contribute to the generation of excitatory and inflammatory mediators, which may participate in regulating the RSNA and increase the risk of VAs in MI rats. Manipulations designed to inhibit HMGB1 activation in the PVN may be an effective method for the present treatment of VAs after MI. These results may provide a fundamental mechanism and therapeutic method for the high incidence of VAs in patients after MI in the future.

\section{Conflict of interest: None declared}

\section{References}

1. Bianchi ME, Beltrame M, Paonessa G. Specific recognition of cruciform DNA by nuclear protein HMG1. Science. 1989; 243(4894 Pt 1): 1056-1059, indexed in Pubmed: 2922595.

2. Ellerman JE, Brown CK, de Vera M, et al. Masquerader: high mobility group box-1 and cancer. Clin Cancer Res. 2007; 13(10): 2836-2848, doi: 10.1158/1078-0432.CCR-06-1953, indexed in Pubmed: 17504981.

3. Ranzato E, Martinotti S, Pedrazzi M, et al. High mobility group box protein-1 in wound repair. Cells. 2012; 1(4): 699-710, doi: 10.3390/cells1040699, indexed in Pubmed: 24710526.

4. Park JS, Svetkauskaite D, He Q, et al. Involvement of toll-like receptors 2 and 4 in cellular activation by high mobility group box 1 protein. J Biol Chem. 2004; 279(9): 7370-7377, doi: 10.1074/ jbc.M306793200, indexed in Pubmed: 14660645.

5. Raman KG, Sappington PL, Yang R, et al. The role of RAGE in the pathogenesis of intestinal barrier dysfunction after hemorrhagic shock. Am J Physiol Gastrointest Liver Physiol. 2006; 291(4): G556-G565, doi: 10.1152/ajpgi.00055.2006, indexed in Pubmed: 16751175.

6. Yang R, Harada T, Mollen KP, et al. Anti-HMGB1 neutralizing antibody ameliorates gut barrier dysfunction and improves survival after hemorrhagic shock. Mol Med. 2006; 12(4-6): 105-114, doi: 10.2119/2006-00010.Yang, indexed in Pubmed: 16953558.

7. Turjanski AG, Vaqué JP, Gutkind JS. MAP kinases and the control of nuclear events. Oncogene. 2007; 26(22): 3240-3253, doi: 10.1038/sj.onc.1210415, indexed in Pubmed: 17496919.

8. Yoon S, Seger R. The extracellular signal-regulated kinase: multiple substrates regulate diverse cellular functions. Growth Factors. 2006; 24(1): 21-44, doi: 10.1080/02699050500284218, indexed in Pubmed: 16393692.
9. Chang F, Steelman LS, Lee JT, et al. Signal transduction mediated by the Ras/Raf/MEK/ERK pathway from cytokine receptors to transcription factors: potential targeting for therapeutic intervention. Leukemia. 2003; 17(7): 1263-1293, doi: 10.1038/ /sj.leu.2402945, indexed in Pubmed: 12835716.

10. Krishna M, Narang H. The complexity of mitogen-activated protein kinases (MAPKs) made simple. Cell Mol Life Sci. 2008; 65(22): 3525-3544, doi: 10.1007/s00018-008-8170-7, indexed in Pubmed: 18668205.

11. Haack KKV, Mitra AK, Zucker IH. NF-kappaB and CREB are required for angiotensin II type 1 receptor upregulation in neurons. PLoS One. 2013; 8(11): e78695, doi: 10.1371/journal. pone.0078695, indexed in Pubmed: 24244341.

12. Mitra AK, Gao L, Zucker IH. Angiotensin II-induced upregulation of AT(1) receptor expression: sequential activation of NFkappaB and Elk-1 in neurons. Am J Physiol Cell Physiol. 2010; 299(3): C561-C569, doi: 10.1152/ajpcell.00127.2010, indexed in Pubmed: 20554912.

13. Coote JH. A role for the paraventricular nucleus of the hypothalamus in the autonomic control of heart and kidney. Exp Physiol. 2005; 90(2): 169-173, doi: 10.1113/expphysiol.2004.029041, indexed in Pubmed: 15604110.

14. Li DP, Pan HL. Glutamatergic inputs in the hypothalamic paraventricular nucleus maintain sympathetic vasomotor tone in hypertension. Hypertension. 2007; 49(4): 916-925, doi: 10.1161/01. HYP.0000259666.99449.74, indexed in Pubmed: 17309953.

15. Badoer E. Role of the hypothalamic PVN in the regulation of renal sympathetic nerve activity and blood flow during hyperthermia and in heart failure. Am J Physiol Renal Physiol. 2010; 298(4): F839-F846, doi: 10.1152/ajprenal.00734.2009, indexed in Pubmed: 20147365.

16. Gao J, Zhong MK, Fan ZD, et al. SOD1 overexpression in paraventricular nucleus improves post-infarct myocardial remodeling and ventricular function. Pflugers Arch. 2012; 463(2): 297-307, doi: 10.1007/s00424-011-1036-0, indexed in Pubmed: 22006090.

17. Han Y, Shi Z, Zhang F, et al. Reactive oxygen species in the paraventricular nucleus mediate the cardiac sympathetic afferent reflex in chronic heart failure rats. Eur J Heart Fail. 2007; 9(10): 967-973, doi: 10.1016/j.ejheart.2007.07.004, indexed in Pubmed: 17719272.

18. Wang HJ, Zhang F, Zhang Y, et al. AT1 receptor in paraventricular nucleus mediates the enhanced cardiac sympathetic afferent reflex in rats with chronic heart failure. Auton Neurosci. 2005; 121(1-2): 56-63, doi: 10.1016/j.autneu.2005.07.003, indexed in Pubmed: 16099221.

19. Zhu GQ, Gao L, Patel KP, et al. ANG II in the paraventricular nucleus potentiates the cardiac sympathetic afferent reflex in rats with heart failure. J Appl Physiol (1985). 2004; 97(5): 1746-1754, doi: 10.1152/japplphysiol.00573.2004, indexed in Pubmed: 15475555.

20. Hu H, Xuan Y, Wang Ye, et al. Targeted NGF siRNA delivery attenuates sympathetic nerve sprouting and deteriorates cardiac dysfunction in rats with myocardial infarction. PLoS One. 2014; 9(4): e95106, doi: 10.1371/journal.pone.0095106, indexed in Pubmed: 24755692.

21. Wang Ye, Xuan YL, Hu HS, et al. Risk of ventricular arrhythmias after myocardial infarction with diabetes associated with sympathetic neural remodeling in rabbits. Cardiology. 2012; 121(1): 1-9, doi: 10.1159/000336148, indexed in Pubmed: 22377967.

22. Chen $\mathrm{AD}$, Xiong $\mathrm{XQ}$, Gan $\mathrm{XB}$, et al. Endothelin- 1 in paraventricular nucleus modulates cardiac sympathetic afferent reflex and sympathetic activity in rats. PLoS One. 2012; 7(7): 
e40748, doi: 10.1371/journal.pone.0040748, indexed in Pubmed: 22815806.

23. Kenney MJ, Weiss ML, Haywood JR. The paraventricular nucleus: an important component of the central neurocircuitry regulating sympathetic nerve outflow. Acta Physiol Scand. 2003; 177(1): 7-15, doi: 10.1046/j.1365-201X.2003.01042.x, indexed in Pubmed: 12492774.

24. Felder RB. Mineralocorticoid receptors, inflammation and sympathetic drive in a rat model of systolic heart failure. Exp Physiol. 2010; 95(1): 19-25, doi: 10.1113/expphysiol.2008.045948, indexed in Pubmed: 19648480.

25. Felder RB, Yu Y, Zhang ZH, et al. Pharmacological treatment for heart failure: a view from the brain. Clin Pharmacol Ther. 2009; 86(2): 216-220, doi: 10.1038/clpt.2009.117, indexed in Pubmed: 19553933.

26. Dardenne AD, Wulff BC, Wilgus TA. The alarmin HMGB-1 influences healing outcomes in fetal skin wounds. Wound Repair Regen. 2013; 21(2): 282-291, doi: 10.1111/wrr.12028, indexed in Pubmed: 23438257.

27. Erlandsson Harris H, Andersson U. Mini-review: The nuclear protein HMGB1 as a proinflammatory mediator. Eur J Immunol. 2004; 34(6): 1503-1512, doi: 10.1002/eji.200424916, indexed in Pubmed: 15162419.

28. Li YF, Patel KP. Paraventricular nucleus of the hypothalamus and elevated sympathetic activity in heart failure: the altered inhibitory mech- anisms. Acta Physiol Scand. 2003; 177(1): 17-26, doi: 10.1046/j.1365201X.2003.01043.x, indexed in Pubmed: 12492775.

29. Brasier AR, Jamaluddin M, Han Y, et al. Angiotensin II induces gene transcription through cell-type-dependent effects on the nuclear factor-kappaB (NF-kappaB) transcription factor. Mol Cell Biochem. 2000; 212(1-2): 155-169, indexed in Pubmed: 11108147.

30. Li XC, Zhuo JL. Nuclear factor-kappaB as a hormonal intracellular signaling molecule: focus on angiotensin II-induced cardiovascular and renal injury. Curr Opin Nephrol Hypertens. 2008; 17(1): 37-43, doi: 10.1097/MNH.0b013e3282f2903c, indexed in Pubmed: 18090668.

31. Siebenlist U, Franzoso G, Brown K. Structure, regulation and function of NF-kappa B. Annu Rev Cell Biol. 1994; 10: 405-455, doi: 10.1146/annurev.cb.10.110194.002201, indexed in Pubmed: 7888182.

32. Tsatsanis C, Androulidaki A, Venihaki M, et al. Signalling networks regulating cyclooxygenase-2. Int J Biochem Cell Biol. 2006; 38(10): 1654-1661, doi: 10.1016/j.biocel.2006.03.021, indexed in Pubmed: 16713323.

33. Yu Y, Wei SG, Zhang ZH, et al. ERK1/2 MAPK signaling in hypothalamic paraventricular nucleus contributes to sympathetic excitation in rats with heart failure after myocardial infarction. Am J Physiol Heart Circ Physiol. 2016; 310(6): H732-H739, doi: 10.1152/ajpheart.00703.2015, indexed in Pubmed: 26801309. 